\title{
Clinical Features, Treatment, and Outcome of Tumor Lysis Syndrome in Germ Cell Tumors
}

\author{
Bryan Leung ${ }^{1}$, Jeffrey Wang ${ }^{2}$, Jill Cassaday ${ }^{3}$ and Jue Wang ${ }^{3,4 *}$ \\ ${ }^{1}$ The University of Arizona College of Pharmacy \\ ${ }^{2}$ Baylor College of Medicine \\ ${ }^{3}$ University of Arizona Cancer Center at Dignity Health St. Joseph's Hospital and Medical Center \\ ${ }^{4}$ University of Arizona College of Medicine - Phoenix \\ *Corresponding author: Jue Wang, MD, FACP, Professor of Medicine, Director of Genitourinary Oncology Section, University of \\ Arizona Cancer Center at Dignity Health St. Joseph's, Phoenix, AZ 85004, Email: jue.wang@dignityhealth.org
}

\begin{abstract}
Background: Tumor lysis syndrome (TLS) is an oncologic emergency that can occur spontaneously or triggered by cancer treatment. This life-threatening condition is most commonly seen in hematologic cancers but is sometimes described in solid tumors such as germ cell tumors (GCTs). The objective of this study is to investigate the clinical characteristics and outcomes of TLS in GCTs.
\end{abstract}

Methods: Retrospective review and pooled analysis.

Result: Seventeen cases of TLS related to GCTs were identified. These included nonseminomatous GCT (7), seminoma (5), choriocarcinoma (2), ovarian (2), and intracranial germinoma (1). Nine cases (53\%) of TLS were treatment-related and eight cases (47\%) occurred spontaneously. The median age of patients was 34 years (range: 13-58), excluding one newborn baby. The median time from treatment to TLS was 2.1 days. Seven patients (41\%) had liver metastases, but all patients had bulky disease. All of the patients received supportive measures; 4 (24\%) patients received rasburicase and 11 patients (64\%) underwent hemodialysis. The mortality rate was $44.4 \%$ among the nine cases of treatment-related TLS, and $37.5 \%$ in the eight patients with spontaneous TLS (STLS).

Conclusion: TLS can occur in GCTs that have a high tumor burden. Almost 50\% of TLS in GCTs happened spontaneously. Considering the life-threatening nature of TLS in GCTs, health care professionals should be alert and monitor for TLS in order to prevent or treat this potentially fatal complication.

Keywords: Tumor lysis syndrome (TLS); Oncologic emergency; Germ cell tumors (GCTs); Clinical features, Treatment, and Outcome; Mortality

\section{Introduction}

Tumor lysis syndrome (TLS) is an oncologic emergency in which tumor cells are lysed, either spontaneously or in response to treatment, releasing their intracellular contents and resulting in metabolic disturbances like hyperuricemia, hyperkalemia, hyperphosphatemia, and hypocalcemia. TLS is most commonly seen in rapidly proliferating tumors like hematologic malignancies. Massive cell death due to chemotherapy and the subsequent rapid release of cytokines potentially contribute to multi-organ failure [1-5]. It is crucial for clinicians to be aware of TLS and to prevent, detect and treat TLS early to prevent life-threatening complications. While cancers are generally seen as diseases of the elderly, germ cell tumors (GCTs) typically affect men between the ages of 18 and 40 . GCTs represent a histologically heterogeneous group of neoplasms derived from the germ-cell lineage. Most tumors occur within the 
gonads; $~ 5 \%$ of GCTs develop at extragonadal locations along the midline of the body $[6,7]$. GCTs are highly proliferative and sensitive to chemotherapy. Even in patients with metastatic disease, cure rates of $80 \%$ can be achieved with multi-agent, cisplatin (CDDP)based combination chemotherapy [7]. As germ-cell tumors are among the fastest growing solid tumors [8] and show an exquisite response to combination chemotherapy, it is not surprising that GCTs are among the few types of solid tumor that have high a risk of TLS. TLS has been noted particularly in patients with bulky disease and highly elevated tumor markers, sometimes showing a fatal outcome $[9,10]$. Currently, there is limited information on the incidence, clinical presentation and outcome of TLS in GCTs. The objective of this case series was to analyze the published case studies on the clinical characteristics, management and results of TLS in patients diagnosed with GCTs.

\section{Methods}

\section{Literature Search Strategy}

Systematic review of the literature was performed by searching PubMed for "germ cell tumor", "testicular cancer", "ovarian cancer", and "tumor lysis syndrome". The identified case studies were reviewed [11-22].

\section{Data Collection and Statistical Analysis}

Information regarding the patient (patient age at diagnosis, presentation and comorbidities), the tumor (symptoms, histology), tumor markers (serum alpha fetoprotein, beta-human chorionic gonadotropin, and lactate dehydrogenase), treatment modalities (chemotherapy), treatment response and outcomes (survival, death) were recorded, when available. Descriptive statistics, such as frequency counts, medians, and ranges, were used to characterize the pooled sample.

\section{Results}

Seventeen publications of TLS (nine cases of treatment-related and eight cases of spontaneous tumor lysis syndrome [STLS]) were identified. The demographic feature, clinicopathologic features and survival outcomes of 17 cases of TLS in GCT were summarized in Table 1 . The median age of patients was 34 years (range: 13-58), excluding the one newborn baby. Male to female ratio was 12:5. The majority of cases were nonseminomatous GCT (7), followed by seminoma (5), choriocarcinoma (2), ovarian (2), and intracranial germinoma (1). Nine cases (53\%) were associated with chemotherapy treatment and 8 cases $(47 \%)$ had STLS. Seven patients (41\%) had extensive liver metastases, but all patients had bulky disease. The median time from treatment to TLS was 2.1 days. All of the patients had elevated LDH, uric acid, creatinine, potassium, and phosphorous. Each of these cases received supportive measures; 4 (24\%) patients received rasburicase and 11 patients (64\%) underwent hemodialysis. The mortality rate for the entire cohort was $41.1 \%$. The mortality rate for patients with and without liver metastases were $57.1 \%$ and $30 \%$, respectively. The mortality rate was $44.4 \%$ among nine cases of treatment-related TLS, with a median survival from diagnosis of TLS to death of 14 days (1-34). The mortality rate was $37.5 \%$ in the eight patients with STLS.

Table 1: Review of published case studies on tumor lysis syndrome in patients with germ cell tumors.

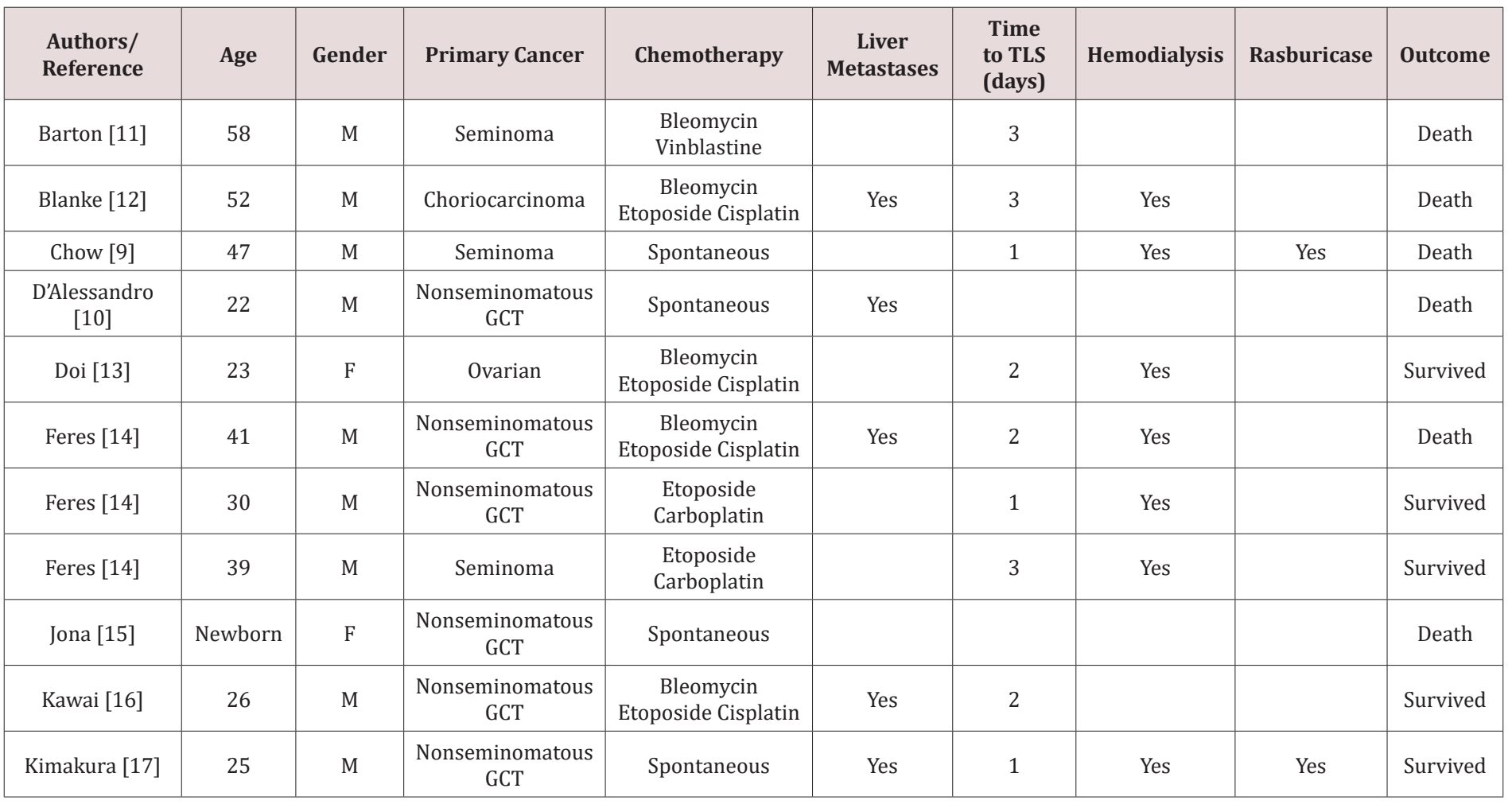




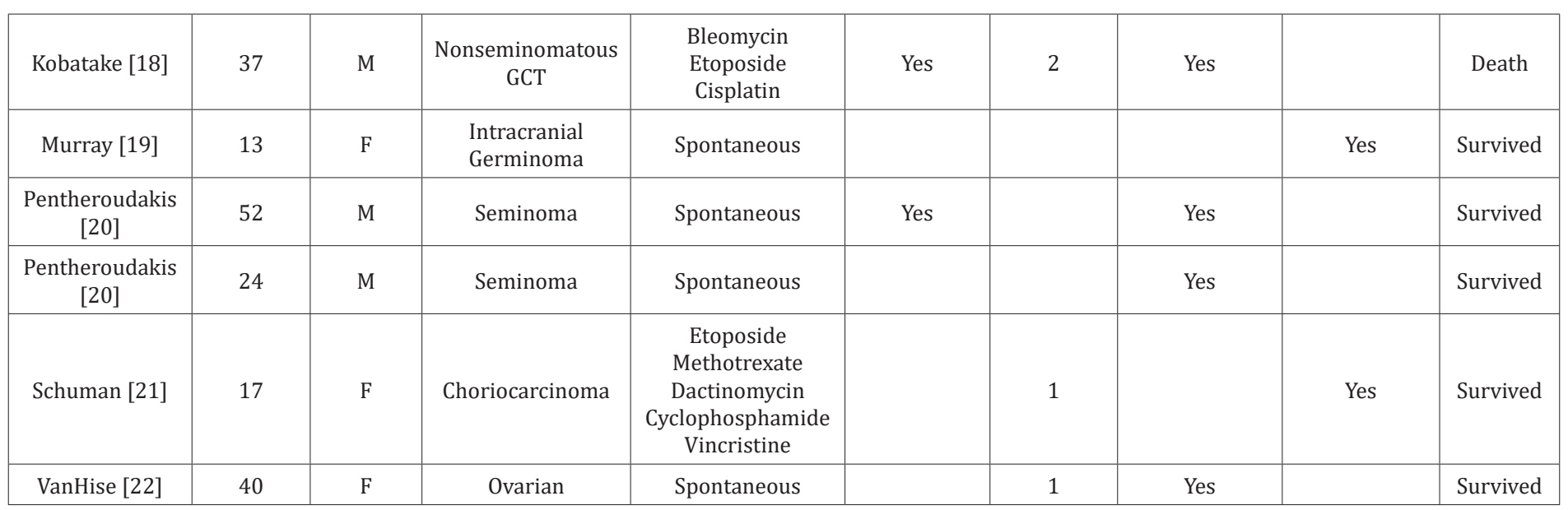

\section{Discussion}

TLS occurs as a result of rapid cancer cell lysis during treatments for hematologic malignancies and select solid tumors. Without aggressive preventive and management measures, TLS can result in serious complications, including death. Historically, solid tumors were considered low risk for developing TLS, whereas TLS was more associated with hematologic cancers. In a study of 132 cases of TLS in solid tumors [1,2], we found that TLS can occur in any type of cancer that has a high tumor burden and is sensitive to chemotherapy or radiotherapy. In our study, we identified 17 cases $(12 \%)$ of TLS in solid tumors that involved GCT. As germcell tumors are among the fastest growing solid tumors [8] and show an exquisite response to combination chemotherapy, it is not surprising that GCTs are among the few solid tumors with a high risk of TLS, especially in patients with bulky disease and highly elevated tumor markers.

GCTs are highly curable and even a majority of patients with metastatic GCTs may expect to be cured [7]. The high cure rates of GCTS are due to their high sensitivity to chemotherapy, which was thought to be related to several factors. Tp53 alteration, a frequent finding in cancers in general, has implications for chemotherapy resistance and is not commonly seen in GCTs [23]. A recent study demonstrated another key to chemo-sensitivity of GCTs appear to lie in their stem cells, which are more sensitive to chemotherapy than stem cells in non-GCT cancers [24]. In Table 2 [25-27], we compare the clinical features and outcomes of TLS in GCTs, renal cell carcinoma, prostate and urothelial cancer. One notable finding is the high rate of spontaneous tumor lysis syndrome (STLS) in GCTs. Almost half of the patients $(8 / 17,47 \%)$ experienced STLS, in contrast to the lower rates of STLS in other solid tumors. The high propensity of STLS in GCTs may be related to the highly proliferative nature of these tumors. Another important finding is the difference in mortality rates among genitourinary tumors. Although GCTs have the most cases of TLS, they have the lowest mortality rate for STLS and treatment-related TLS amongst these four cancers; a total of 7 patients (41\%) with GCTs died after being diagnosed with TLS. One possible reason for the lower mortality of TLS in GCTs is that patients with GCTs are generally younger than patients with other genitourinary tumors. The median age of patients with GCTs and TLS was about half that of patients with other genitourinary tumors and TLS. Older age is associated with more comorbidities, likely contributing to the higher mortality rate in non-germ cell genitourinary tumors. Secondly, the increased awareness of TLS risk in patients with GCTs may contribute to better prognosis. On the other hand, physicians do not routinely consider TLS as a diagnostic possibility for non-germ cell genitourinary tumors nor treat prophylactically. Nevertheless, the mortality rate of TLS in GCTs remains unacceptable and further research into pathophysiology of fatal TLS is warranted.

Table 2: Review of tumor lysis syndrome in patients with germ cell tumor, renal cell carcinoma, prostate cancer, and urothelial cancer.

\begin{tabular}{|c|c|c|c|c|c|c|}
\hline Cancer & $\begin{array}{c}\text { Median Age } \\
\text { (Range) }\end{array}$ & Total & Spontaneous TLS & $\begin{array}{c}\text { Treatment- } \\
\text { Related TLS }\end{array}$ & $\begin{array}{c}\text { Mortality from } \\
\text { STLS }\end{array}$ & $\begin{array}{c}\text { Mortality from } \\
\text { Treatment- } \\
\text { Related TLS }\end{array}$ \\
\hline Germ Cell Tumor & $34(13-58)$ & 17 & $8(47 \%)$ & $9(53 \%)$ & $3 / 8(37.5 \%)$ & $4 / 9(44.4 \%)$ \\
\hline $\begin{array}{c}\text { Renal Cell } \\
\text { Carcinoma [3] }\end{array}$ & $60(45-88)$ & 10 & $3(30 \%)$ & $7(70 \%)$ & $3 / 3(100 \%)$ & $4 / 7(57 \%)$ \\
\hline Prostate [4] & $69(53-80)$ & 14 & $8(57.1 \%)$ & $6(42.9 \%)$ & $3 / 8(37.5 \%)$ & $6 / 6(100 \%)$ \\
\hline Urothelial [25-27] & $73(67-77)$ & 3 & $0(0 \%)$ & $3(100 \%)$ & - & $2 / 3(66.7 \%)$ \\
\hline
\end{tabular}


Our case series highlights a rare but potential life-threatening oncological emergency of GCTs. As there will never be randomized trials elucidating the optimal management of TLS, our findings underscore the importance of real-world data to provide a better understanding of the prevention and treatment of this complication. It is our hope that the information presented here can raise awareness among community physicians to the detection and management of TLS in GCTs. Since all fatal TLS occurred during the first cycle of chemotherapy, a dose modification in the first cycle of chemotherapy can be considered in the high risks of TLS. However, the optimal management of TLS will require a better understanding of the underlying pathophysiology of the lethal form of TLS.

\section{Conclusion}

Our current case series consists of the largest reported cohort of TLS in GCTs. Our study provides the most current information regarding the clinical features and outcome of TLS in GCTs. There is an urgent need for better understanding of lethal TLS and for the development of optimal multidisciplinary management strategies for this rare, but potentially fatal oncologic emergency.

\section{References}

1. Wang JF, Harmon J, Allen S, Cassaday J, Ruvinov K, et al. (2015) Older age and liver metastases predict poor prognosis of Tumor Lysis Syndrome in Solid Tumor. In: Wang J (Eds.), Top 25 Contributions on Cancer Research. Avid Science Borsigstr. 9, 10115 Berlin, Berlin, Germany.

2. Harmon J, Allen S, Cassaday J, Ruvinov K, Stoyanova D (2018) Liver Metastasis is an Independent Predictor for Mortality in Patients Who Developed Tumor Lysis Syndrome: Analysis of 132 Patients with Solid Tumors. J Clin Oncol 36(15s): e18766.

3. Allen S, William A, Cassaday J, Wang J (2018) Renal Cell Carcinoma with Extensive Rhabdoid Features: Case Report of Spontaneous Tumor Lysis Syndrome and Review of Literature. Urol Res Ther J 2(1): 119.

4. Wang J (2018) Tumor Lysis Syndrome Associated with Prostate Cancer: An Under-recognized Oncologic Emergency. J Emerg Crit Care Diagn Manag 1(1): 1-3.

5. Harmon J, Allen S, Cassaday J, Ruvinov K, Stoyanova D (2018) The Clinical Features, Treatment, Outcome and Prognosis of Spontaneous Tumor Lysis Syndrome in Solid Tumor. J Oncol Res Forecast 1(2): 1009.

6. Hainsworth JD, Greco FA (1992) Extragonadal germ cell tumors and unrecognized germ cell tumors. Semin Oncol 19(2): 119-127.

7. Motzer RJ, Agarwal N, Beard C, Bolger GB, Boston B (2009) NCCN Clinical Practice Guidelines in Oncology: Testicular Cancer. J Natl Compr Canc Netw 7(6): 672-693.

8. Charbit A, Malaise E, and Tubiana M (1971) Relation between the pathological nature and the growth rate of human tumors. European Journal of Cancer 7(4): 307-315.

9. Chow M, Yuwono A, Tan R (2016) Tumour lysis syndrome: A rare acute presentation of locally advanced testicular cancer-Case report and review of literature. Asian Journal of Urology 3(1): 49-52.

10. D Alessandro V, Greco A, Clemente C (2019) Severe Spontaneous Acute Tumor Lysis Syndrome and Hypoglycemia in Patient with Germ Cell Tumor. Tumori Journal 96(6): 1040-1043.
11. Barton J (1989) Tumor lysis syndrome in nonhematopoietic neoplasms. Cancer 64(3): 738-740.

12. Blanke C, Hemmer P and Witte R (2000) Acute Tumor Lysis Syndrome with Choriocarcinoma. South Med J 93(9): 916-919.

13. Doi M, Okamoto Y, Yamauchi M (2011) Bleomycin-induced pulmonary fibrosis after tumor lysis syndrome in a case of advanced yolk sac tumor treated with bleomycin, etoposide and cisplatin (BEP) chemotherapy. International Journal of Clinical Oncology 17(5): 528-531.

14. Feres G, Soares M, Salluh J (2008) Severe acute tumor lysis syndrome in patients with germ-cell tumors. Indian Journal of Urology 24(4): 555557.

15. Jona J (1999) Progressive Tumor Necrosis and Lethal Hyperkalemia in a Neonate with Sacrococcygeal Teratoma (SCT) Journal of Perinatology 19(7): 538-540.

16. Kawai K, Takaoka E, Naoi M (2006) A Case of Metastatic Testicular Cancer Complicated by Tumour Lysis Syndrome and Choriocarcinoma Syndrome. Japanese Journal of Clinical Oncology 36(10): 665-667.

17. Kimakura M, Abe T, Nagahara A (2016) Metastatic testicular cancer presenting with liver and kidney dysfunction treated with modified BEP chemotherapy combined with continuous hemodiafiltration and rasburicase. Anti-Cancer Drugs 27(4): 364-368.

18. Kobatake K, Kato M, Mita K (2015) Advanced testicular cancer associated with life-threatening tumour lysis syndrome and choriocarcinoma syndrome. Canadian Urological Association Journal 9(1-2): 62.

19. Murray M, Metayer L, Mallucci C (2011) Intra-abdominal metastasis of an intracranial germinoma via ventriculo-peritoneal shunt in a 13-yearold female. British Journal of Neurosurgery 25(6): 747-749.

20. Pentheroudakis G, O Neill V, Vasey P (2001) Spontaneous acute tumour lysis syndrome in patients with metastatic germ cell tumours. Supportive Care in Cancer 9(7): 554-557.

21. Schuman S, Pearson J, Lucci J (2010) Metastatic gestational trophoblastic neoplasia complicated by tumor lysis syndrome, heart failure, and thyrotoxicosis: a case report. The Journal of Reproductive Medicine 55(9-10): 441-444.

22. VanHise K, Swailes A, Roche M (2017) Tumor lysis syndrome in a patient with ovarian yolk sac tumor. Gynecologic Oncology Reports 22: 92-93.

23. Lutzker SG (1998) p53 tumour suppressor gene and germ cell neoplasia. APMIS 106: 85-89.

24. Pierpont TM, Lyndaker AM, Anderson CM (2017) ChemotherapyInduced Depletion of OCT4-Positive Cancer Stem Cells in a Mouse Model of Malignant Testicular Cancer. Cell Rep 21(7): 1896-1909.

25. Wang J, Ruvinov K, Cassaday J (2017) Acute Tumor Lysis Syndrome after Cisplatin and Gemcitabine for Treatment of Urothelial Carcinoma of the Renal Pelvis: Case Report and Review of Literature. Ann Clin Lab Res 5(2): 165-168.

26. Lin CJ, Lim KH, Cheng YC, Chen HH, Wu CJ (2007) Tumor lysis syndrome after treatment with gemcitabine for metastatic transitional cell carcinoma. Med Oncol 24(4): 455-457.

27. Brunnhoelzl D, Weed M, Trepet R, Wang J (2017) Tumor Lysis Syndrome Following a Single Atezolizumab Infusion for Metastatic Urothelial Carcinoma Involving both Upper and Lower Tract. Archives in Cancer Research 5(1): 127. 
To Submit Your Article Click Here: Submit Article

DOI: $10.32474 / J U N S .2019 .01 .000116$

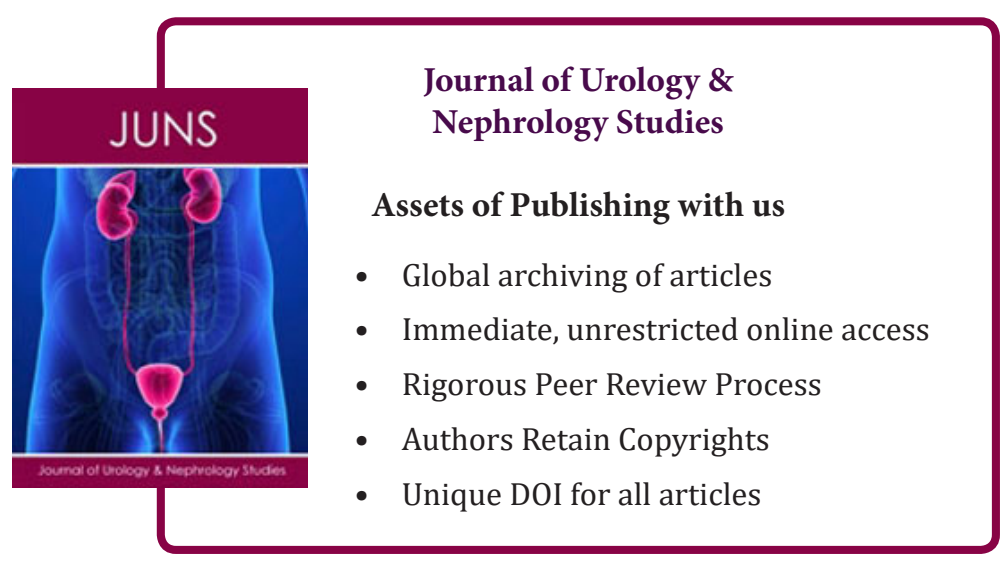

